\title{
Analysis of the energy decay of a viscoelasticity type equation
}

\author{
Amel Atallah-Baraket and Maryem Trabelsi
}

\begin{abstract}
In this paper, we study the evolution of the energy density of a sequence of solutions of a problem related to a viscoelasticity model where the viscosity term is a pseudo-differential operator of order $2 \alpha$ with $\alpha \in(0,1)$. We calculate the weak limit of the energy density in terms of microlocal defect measures and under special assumption we prove that the viscosity term prevents propagation of concentration and oscillation effects contrary to what happens in the wave equation.
\end{abstract}

\section{Introduction}

We consider the equation of viscoelasticity given by

$$
\left\{\begin{array}{l}
\partial_{t}^{2} u-\nabla \cdot(C(x) \nabla u)+q(x, D)^{*} q(x, D) \partial_{t} u=0, \quad(t, x) \in \mathbb{R}_{+} \times \Omega, \\
u_{\mid t=0}=u_{0} \\
\partial_{t} u_{\mid t=0}=u_{1} \\
u_{\mid \partial \Omega}=0
\end{array}\right.
$$

Key Words: viscoelasticity equation, microlocal defect measures, energy density 2010 Mathematics Subject Classification: Primary 46G05, 46L05; Secondary 47A30, $47 \mathrm{~B} 47$.

Received: 16.01 .2016

Accepted: 16.02 .2016 
where $\Omega$ is an open subset of $\mathbb{R}^{d}, u$ a scalar real-valued function, $C(x)$ is a symmetric positive matrix and is supposed to depend smoothly on the variable $x \in \Omega$. Moreover we suppose that there exists $C_{1}, C_{2}>0$ such that

$$
\forall x \in \Omega, \forall \xi \in \mathbb{R}^{d}, \quad C_{1}|\xi|^{2} \leq C(x) \xi . \xi \leq C_{2}|\xi|^{2}
$$

$q(x, D)$ is a pseudo-differential operator defined by

$$
q(x, D) f(x)=\int_{\Omega \times \mathbb{R}^{d}} q(x, \xi) e^{i \xi \cdot(x-y)} f(y) d y d \xi, \quad \forall f \in \mathcal{S}\left(\mathbb{R}^{d}\right)
$$

such as his symbol $q(x, \xi)$ is a classical symbol of order $\alpha$, with $\alpha \in(0,1)$ and $q(x, D)^{*}$ his adjoint operator. This equation has an energy given by

$$
E(t):=\int_{\Omega}\left|\partial_{t} u(t, x)\right|^{2} d x+\int_{\Omega} C(x) \nabla u(t, x) \cdot \nabla u(t, x) d x
$$

which decreases in time according to

$$
E(t)-E(0)=-2 \int_{0}^{t}\left\|q(., D) \partial_{t} u(s, .)\right\|_{L^{2}(\Omega)}^{2} d s \leq 0 .
$$

Equation (4) gives a priori estimates for initial data $u_{0} \in H^{1}(\Omega)$ and $u_{1} \in$ $L^{2}(\Omega)$ and yields by classical arguments the existence of a unique solution $u \in C^{0}\left(\mathbb{R}_{+}, \dot{H}^{1}(\Omega)\right) \cap C^{1}\left(\mathbb{R}_{+}, L^{2}(\Omega)\right)$.

We consider sequences $\left(u^{n}\right)_{n}$ of solutions to (1) with initial data $\left(u_{0}^{n}\right)_{n}$, $\left(u_{1}^{n}\right)_{n}$ which are uniformly bounded in $H^{1}(\Omega)$ and $L^{2}(\Omega)$ respectively. Then, because of (2) and (4), for all $T \geq 0$, the families $\left(\nabla u^{n}(T)\right)_{n}$ and $\left(\partial_{t} u^{n}(T)\right)_{n}$ are uniformly bounded in $L^{2}(\Omega)$ and $\left(q(x, D) \partial_{t} u^{n}\right)_{n}$ is uniformly bounded in $L^{2}\left([0, T], L^{2}(\Omega)\right)$.

Our aim is to describe the evolution of the weak limit in the set of measures of the energy density $e^{n}(t,$.

$$
\forall t \in \mathbb{R}_{+}, e^{n}(t, x)=\left[\left|\partial_{t} u^{n}(t, x)\right|^{2}+C(x) \nabla u^{n}(t, x) \cdot \nabla u^{n}(t, x)\right] d x .
$$

Without loss of generality, we suppose that $\left(u_{0}^{n}\right)_{n}$ and $\left(u_{1}^{n}\right)_{n}$ goes to 0 weakly in $H^{1}(\Omega)$ and $L^{2}(\Omega)$ respectively.

In the case where $\Omega=\mathbb{R}^{d}, q(x, \xi)=|\xi|^{\alpha},|\xi| \geq 1$, and $C=c I_{d}, c>0$, where $I_{d}$ is the identity matrix of order $\mathrm{d}$, using the Fourier transform of a family of solutions of (1), we get a differential linear equation of order 2 in the variable $t$. Solving this equation gives that for $t>0$ and for $\alpha \in(1 / 2,1)$, 


$$
\widehat{u^{n}}(t, \xi) \underset{|\xi| \rightarrow+\infty}{\longrightarrow} 0 .
$$

Our aim is to generalize this result to the case where $C$ depend on the variable $x$ and $q$ is a classical symbol of order $\alpha$ with $\alpha \in(0,1)$.

The description of the energy decay has been the subject of several contributions. In particular in [13], Francfort and Murat, have proved that for the wave equation, there is a propagation of the energy density in the phase space (see also [8]). Our aim is close to that of Atallah and Fermanian who studied in [2] the case of $\alpha=1$. Specifically they studied the evolution of the energy density of a family of solutions to the Kelvin-Voigt viscoelasticity equation given by

$$
\left\{\begin{array}{l}
\partial_{t}^{2} u-\nabla \cdot(C(x) \nabla u)-\nabla \cdot\left(B(x) \nabla \partial_{t} u\right)=0, \quad(t, x) \in \mathbb{R}_{+} \times \Omega \\
u_{\mid t=0}=u_{0} \in H_{0}^{1}(\Omega) \\
\partial_{t} u_{\mid t=0}=u_{1} \in L^{2}(\Omega) \\
u_{\mid \partial \Omega}=0
\end{array}\right.
$$

where $\Omega$ is an open subset of $\mathbb{R}^{d}$ and $u$ a scalar real-valued function. Matrices $C(x)$ and $B(x)$ are symmetric, non-negative and are supposed to depend smoothly on the variable $x \in \Omega$. Moreover, they supposed that $C(x)$ is a positive matrix for which there exists $C_{1}, C_{2}>0$ such that

$$
\forall x \in \Omega, \forall \xi \in \mathbb{R}^{d}, \quad C_{1}|\xi|^{2} \leq C(x) \xi . \xi \leq C_{2}|\xi|^{2} .
$$

The matrix $B$ is also assumed to be positive and satisfies

$$
\forall x \in \Omega, \forall \xi \in \mathbb{R}^{d}, \quad 0 \leq B(x) \xi . \xi \leq C_{3}|\xi|^{2}, \quad C_{3}>0 .
$$

They proved under special assumptions that the oscillation or concentration effects do not propagate but are damped in time. Also G. Lebeau has studied in [19] the case $\alpha=0$, more precisely he considered the equation of damped waves given by

$$
\left\{\begin{array}{l}
\left(\partial_{t}^{2}-\Delta+2 a(x) \partial_{t}\right) u=0, \\
u_{\mid \mathbb{R}_{t} \times \partial M} \equiv 0 \\
u_{\mid t=0}=u_{0} \in H_{0}^{1}(M), \\
\partial_{t} u_{\mid t=0}=u_{1} \in L^{2}(M),
\end{array}\right.
$$

where $M$ is a compact riemannian manifold.

This equation has an energy given by

$$
E(u, t):=\frac{1}{2} \int_{M}\left|\partial_{t} u(t, x)\right|^{2} d x+\int_{M}\left|\nabla_{x} u\right|^{2} .
$$


He proved that for all $u_{0} \in H_{0}^{1}(M)$ and $u_{1} \in L^{2}(M)$, we have

$$
E(u, t) \underset{t \rightarrow \infty}{\longrightarrow} 0
$$

Moreover he also proved that under some geometrical conditions on the manifold we have exponential decay of the energy.

For more results on the viscoelasticity equations, the reader can refers to $[3,5,10,18,21]$. To calculate the weak limit of the energy density, we need to express the limit of quadratic products of weakly oscillating quantities. For this purpose, we use the formalism of microlocal defect measures also called H-measures introduced independently by P. Grard and L. Tartar (see [14] and [24]). We also refer to the books [16] and [23] for related abstract results and applications.

\section{Main Results}

In this section, we present our results which crucially rely on the use of microlocal defect measures that we define in the first subsection. In the second subsection we discuss the evolution of the energy density which is a corollary of the analysis of the behavior of microlocal defect measures associated to the sequences $\left(\partial_{t} u^{n}\right)_{n}$ and $\left(\nabla u^{n}\right)_{n}$.

\subsection{Microlocal defect measures}

Microlocal defect measures allow to treat quadratic quantities like energy density by taking into account microlocal effects. They describe up to a subsequence the limit of quantities of the form $\left(a(x, D) u^{n}, u^{n}\right)_{n}$ where $a(x, D)$ is a pseudo-differential operator and $\left(u^{n}\right)_{n}$ a uniformly bounded family of $H^{s}(\Omega)$. We denote by $\Sigma^{m}(\Omega)$ the set of symbols of order $m, m \in \mathbb{R}$ on $\Omega \times \mathbb{R}^{d}$, i.e. of smooth functions $a \in C^{\infty}\left(\Omega \times \mathbb{R}^{d}\right)$ compactly supported in the variable $x$ in $\Omega$ and satisfying for all multi-indices $\alpha=\left(\alpha_{1}, \ldots, \alpha_{d}\right), \beta=\left(\beta_{1}, \ldots, \beta_{d}\right)$

$$
\left|\partial_{x}^{\alpha} \partial_{\xi}^{\beta} a(x, \xi)\right| \leq C_{\alpha, \beta}\langle\xi\rangle^{m-|\beta|},
$$

where $\partial_{x}^{\alpha}=\partial_{x_{1}}^{\alpha_{1}} \cdots \partial_{x_{d}}^{\alpha_{d}}, \partial_{\xi}^{\beta}=\partial_{\xi_{1}}^{\beta_{1}} \cdots \partial_{\xi_{d}}^{\beta_{d}}$ and $\langle\xi\rangle=\left(1+|\xi|^{2}\right)^{1 / 2}$. Let $a \in \Sigma^{m}(\Omega)$, the symbol $a$ is said to be a classical symbol if there exists a sequence of functions $\left(a_{m-j}\right)_{j \in \mathbb{N}}$ homogeneous of degree $m-j$ for $|\xi| \geq 1$, 
such that for all $k \in \mathbb{N}$,

$$
\left(a-\sum_{j=0}^{k} a_{m-j}\right) \in \Sigma^{m-k-1}(\Omega)
$$

We write $a \in \Sigma_{c}^{m}(\Omega), a \sim \sum_{j=0}^{+\infty} a_{m-j}$ and $a_{m}$ is called the principal symbol of $a$.

Then the operator $a(x, D)$ is defined by

$$
a(x, D) u(x)=\int_{\Omega \times \mathbb{R}^{d}} a(x, \xi) e^{i \xi \cdot(x-y)} u(y) d y d \xi
$$

The operator $a(x, D)$ maps $H^{s}$ into $H^{s-m}$ for all $s \in \mathbb{R}$ (see[1]). Observe that even though the symbol $a$ is positive, the operator $a(x, D)$ is not a positive operator. We recall here an inequality of Gårding called " Low Inequality of Gårding" which connect the positivity of a symbol to that of the operator:

Lemma 1. If $a \in \Sigma^{2 m+1}(\Omega)$, Re $a \geq 0$, then there exist $C>0$ such that

$$
\operatorname{Re}(a(x, D) u, u)) \geq-C\|u\|_{H^{m}}^{2} .
$$

Let $\left(u^{n}\right)$ a uniformly bounded sequence in $H^{m}(\Omega)$ which converges weakly to 0 in $H^{m}$. Then there exists a subsequence $n_{k}, n_{k} \underset{k \rightarrow \infty}{\longrightarrow}+\infty$, and a positive Radon measure $\mu$ on $\Omega \times S^{d-1}$ such that

$$
\forall a \in \Sigma_{c}^{2 m}(\Omega),\left(a(x, D) u^{n_{k}}, u^{n_{k}}\right) \underset{k \rightarrow \infty}{\longrightarrow}\left\langle a_{2 m}, \mu\right\rangle .
$$

Such a measure $\mu$ is called a $H^{m}$-microlocal defect measure of the family $\left(u^{n}\right)_{n}$. One can observe that the positivity of the defect measure is a consequence of the Gårding inequality above. In the following, we denote by $\mathcal{M}^{+}\left(\Omega \times S^{d-1}\right)$ the set of positive Radon measures on $\Omega \times S^{d-1}$.

\subsection{Evolution of the energy density}

Let $\mu_{0}$ be a $H^{1}$-microlocal defect of $\left(u_{0}^{n}\right)_{n}$ and $\lambda_{0}$ a $L^{2}$-microlocal defect measure of $\left(u_{1}^{n}\right)_{n}$. For simplicity, we suppose that $\left(u_{0}^{n}\right)_{n}$ (respectively. $\left.\left(u_{1}^{n}\right)_{n}\right)$ have only one microlocal defect measure. Then, for $a \in \Sigma_{c}^{2}$ (respectively $a \in \Sigma_{c}^{0}$ ) all 
the limit points of $\left(a(x, D) u_{0}^{n} \mid u_{0}^{n}\right)$ (respectively $\left.\left(a(x, D) u_{1}^{n} \mid u_{1}^{n}\right)\right)$ are the same. Therefore we have for $t=0, \forall \phi \in C_{0}^{\infty}(\Omega)$

$$
\begin{array}{r}
\int_{\Omega} \phi(x) e^{n}(0, x) d x \underset{n \rightarrow+\infty}{\longrightarrow} \int_{\Omega \times S^{d-1}} \phi(x) C(x) \omega \cdot \omega \mu_{0}(d x, d \omega) \\
+\int_{\Omega \times S^{d-1}} \phi(x) \lambda_{0}(d x, d \omega) .
\end{array}
$$

\section{Notations:}

1. We will use the abbreviation "u.b." for uniformly bounded.

2. We denote by $\Lambda$ the subset of $\Omega \times S^{d-1}$ defined by

$$
\Lambda:=\left\{(x, \omega) \in \Omega \times S^{d-1}, q_{\alpha}(x, \omega)=0\right\},
$$

where $q_{\alpha}$ is the principal symbol of $q$ and we suppose that $\Lambda \neq \Omega \times S^{d-1}$.

3. We will use special symbols which will satisfy the two following properties:

(i) $a \in \Sigma^{m}(\Omega)$.

(ii) there exists a neighborhood $\mathcal{V}$ of $\Lambda$ such that

$$
a(x, \xi)=0, \forall\left(x, \frac{\xi}{|\xi|}\right) \in \mathcal{V} .
$$

We will denote by $\Sigma_{\Lambda}^{m}$ the class of such symbols.

By the analysis of microlocal defect measures of $\left(\nabla u^{n}(t)\right)_{n}$ and $\left(\partial_{t} u^{n}(t)\right)_{n}$, we will prove the following result for the densities $e^{n}$.

Theorem 1. Suppose that $C$ is smooth and satisfies (2). Suppose moreover that $\mu_{0}(\Lambda)=\lambda_{0}(\Lambda)=0$ and that $\Lambda$ is bounded, then for any $\phi \in C_{0}^{\infty}(\Omega)$ and for every $t>0$

$$
\int_{\Omega} \phi(x) e^{n}(t, x) d x \underset{n \rightarrow+\infty}{\longrightarrow} 0 .
$$

\section{Remark 1 .}

1) If $\lambda_{0} \neq 0$ or $\mu_{0} \neq 0$ and if we denote by $e(t, x)$ the weak limit of the density $e^{n}(t, x)$, the map $t \mapsto e(t,$.$) is discontinuous in t=0$.

2) The oscillation or concentration effects do not propagate. We recall that in the case of the wave equation there is propagation of microlocal defect measures in the phase space (see $[8,13])$. Then the viscoelastic term $q(x, D)^{*} q(x, D) \partial_{t} u$ is predominant in (1) against the wave type term $\nabla \cdot(C(x) \nabla u)$. 
Since our purpose is to calculate the weak limit of the energy density, we get using microlocal defect measures that for any $\phi \in C_{0}^{\infty}(\Omega)$ and for every $t>0$, there exist a subsequence $n_{k}(t)$ such that

$$
\int_{\Omega} \phi(x) e^{n_{k}(t)}(t, x) d x \underset{k \rightarrow+\infty}{\longrightarrow} \int_{\Omega \times S^{d-1}} \phi(x)(\tilde{\lambda}(t, d x, d \omega)+C(x) \omega \cdot \omega \tilde{\mu}(t, d x, d \omega)),
$$

where $\tilde{\lambda}$ (respectively $\tilde{\mu}$ ) is a $L^{2}$-microlocal defect measure of the family $\left(\partial_{t} u^{n_{k}(t)}\right)_{k}$ (respectively $\tilde{\mu}$ is a $H^{1}$-microlocal defect measure of the family $\left.\left(u^{n_{k}(t)}\right)_{k}\right)$. Then the dependence on $t$ of the extracted subsequence of the result (12) is an issue. To overcome this difficulty, we use other measures. Indeed, since

$$
u^{n} \in L_{l o c}^{2}\left(\mathbb{R}, H^{1}(\Omega)\right) \text { and } \partial_{t} u^{n} \in L_{l o c}^{2}\left(\mathbb{R}, L^{2}(\Omega)\right),
$$

then for any $T>0$, there exists a subsequence $m_{k}(T)$ and two measures $\mu(t, x, \omega)$ and $\lambda(t, x, \omega)$ in $L^{\infty}\left([0, T], \mathcal{M}^{+}\left(\Omega \times S^{d-1}\right)\right)$, such that $\forall a \in \Sigma_{c}^{0}(\Omega)$, $\forall \psi \in C_{0}^{\infty}([0, T]), \forall i, j \in\{1, \ldots, d\}$,

$$
\begin{aligned}
\int_{0}^{T} & \psi(t)\left(a(x, D) \partial_{x_{i}} u^{m_{k}(T)}(t) \mid \partial_{x_{j}} u^{m_{k}(T)}(t)\right) d t \\
& \underset{k \rightarrow+\infty}{\longrightarrow} \int_{[0, T] \times \Omega \times S^{d-1}} \psi(t) a_{0}(x, \omega) \omega_{i} \omega_{j} \mu(t, d x, d \omega) d t
\end{aligned}
$$

and

$$
\begin{aligned}
& \int_{0}^{T} \psi(t)\left(a(x, D) \partial_{t} u^{m_{k}(T)}(t) \mid \partial_{t} u^{m_{k}(T)}(t)\right) d t \\
& \underset{k \rightarrow+\infty}{\longrightarrow} \int_{[0, T] \times \Omega \times S^{d-1}} \psi(t) a_{0}(x, \omega) \lambda(t, d x, d \omega) d t .
\end{aligned}
$$

Then for any $T>0$, there exists a subsequence $m_{k}(T)$ such that $\forall \phi \in C_{0}^{\infty}(\Omega)$ and $\forall \psi \in C_{0}^{\infty}([0, T])$

$$
\begin{aligned}
& \int_{[0, T] \times \Omega} \psi(t) \phi(x) e^{m_{k}(T)}(t, x) d x d t \\
& \underset{n \rightarrow+\infty}{\longrightarrow} \int_{[0, T] \times \Omega \times S^{d-1}} \psi(t) \phi(x)(\lambda(d x, d \omega)+C(x) \omega \cdot \omega \mu(d x, d \omega) d t),
\end{aligned}
$$

One can observe that the dependence on $t$ of the extracted subsequence in (12) implies that if $\tilde{\lambda}(t)$ (respectively $\tilde{\mu}(t)$ ) is a $L^{2}$-microlocal defect measure of the family $\left(\partial_{t} u^{n}(t)\right)_{n}$ (respectively is a $H^{1}$-microlocal defect measure of the family $\left.\left(u^{n}(t)\right)_{n}\right)$, then the measures $\lambda(t)$ (respectively $\left.\mu(t)\right)$ and $\tilde{\lambda}(t)$ respectively $\tilde{\mu}(t))$ are not necessary the same. 
Proposition 1. If $\mu_{0}(\Lambda)=\lambda_{0}(\Lambda)=0$ and if $\Lambda$ is bounded, then for $t>0$ the sequence $\left(\partial_{t} u^{n}(t)\right)_{n}\left(\right.$ resp. $\left.\left(u^{n}(t)\right)_{n}\right)$ goes to 0 strongly in $L_{l o c}^{2}(\Omega)\left(\right.$ resp. $\left.H_{l o c}^{1}(\Omega)\right)$.

If we suppose that Proposition 1 holds then by (12), we get Theorem 1. This Proposition will be proved in Section 3.

On the other hand, since $\left(q(x, D) \partial_{t} u^{n}\right)_{n}$ is u.b. in $L^{2}\left([0, T], L^{2}(\Omega)\right)$ then the microlocal defect measure of this sequence describes the limit of the quantity

$$
\int_{\mathbb{R}_{+} \times \Omega} \chi(t) \phi(x)\left(q(x, D) \partial_{t} u^{n}\right)^{2} d t d x
$$

for any $\chi \in C_{0}^{\infty}\left(\mathbb{R}_{+}^{*}\right)$ and $\phi \in C_{0}^{\infty}(\Omega)$. In Proposition 2 below we prove that the knowledge of $\mu$ and $\lambda$ is enough to calculate such a limit.

Proposition 2. Let $\chi \in C_{0}^{\infty}\left(\mathbb{R}_{+}^{*}\right)$ and $\phi \in C_{0}^{\infty}(\Omega)$,

$$
\int_{\mathbb{R}_{+} \times \Omega} \chi(t) \phi(x)\left(q(x, D) \partial_{t} u^{n}\right)^{2} d t d x \underset{n \rightarrow+\infty}{\longrightarrow} 0 .
$$

This result is proved in the end of Section 3.

\section{Proof of the main results}

\subsection{Preliminaries.}

We state technical results that we will use in the next subsections.

Proposition 3. $\forall T>0, \forall a \in \Sigma_{\Lambda}^{0},\left(a(x, D) \partial_{t} u^{n}\right)_{n}$ is uniformly bounded in $L^{2}\left([0, T], H^{\alpha}(\Omega)\right)$.

Proof. Let $a \in \Sigma_{\Lambda}^{0}$ and $\psi$ a cut-off function compactly supported in $\mathbb{R}^{d}$, $0 \leq \psi \leq 1$, and $\psi=1$ near 0 . We write

$$
a(x, \xi)=\psi\left(\frac{\xi}{R}\right) a(x, \xi)+\left(1-\psi\left(\frac{\xi}{R}\right)\right) a(x, \xi) .
$$

We set

$$
\tilde{a}(x, \xi)=\frac{\left(1-\psi\left(\frac{\xi}{R}\right)\right) a(x, \xi)}{q_{\alpha}(x, \xi)} .
$$


If $(x, \xi) \in \operatorname{supp}\left(\left(1-\psi\left(\frac{\xi}{R}\right)\right) a(x, \xi)\right)$ then we have $q_{\alpha}(x, \xi) \neq 0$.

Therefore

$$
\tilde{a}(x, \xi) \in \Sigma_{\Lambda}^{-\alpha}
$$

This gives

$$
\left(1-\psi\left(\frac{\xi}{R}\right)\right) a(x, \xi)=\frac{\left(1-\psi\left(\frac{\xi}{R}\right)\right) a(x, \xi)}{q_{\alpha}(x, \xi)} q(x, \xi)+r(x, \xi),
$$

with $r \in \Sigma^{-1}(\Omega)$.

So, we obtain the following decomposition

$$
a(x, D)=\tilde{a}(x, D) q(x, D)+k(x, D)+r(x, D),
$$

with $k(x, D)$ a compact operator of $L^{2}(\Omega)$. Since $\left(q(x, D) \partial_{t} u^{n}\right)_{n}$ is u.b. in $L^{2}\left([0, T], L^{2}(\Omega)\right)$ and $\tilde{a}(x, \xi) \in \Sigma_{\Lambda}^{-\alpha}$, then $\left(\tilde{a}(x, D) q(x, D) \partial_{t} u^{n}\right)_{n}$ is u.b in $L^{2}\left([0, T], H^{\alpha}(\Omega)\right)$. Besides, since $\left(\partial_{t} u^{n}\right)_{n}$ is u.b. in $L^{2}\left([0, T], L^{2}(\Omega)\right)$ then $\left(r(x, D) \partial_{t} u^{n}\right)_{n}$ is u.b in $L^{2}\left([0, T], H^{1}(\Omega)\right)$, which gives Proposition 3 .

We will often make use of the following Rellich Lemma.

Lemma 2. Let $\left(f_{n}\right)_{n}$ and $\left(g_{n}\right)_{n}$ be two sequences which are uniformly bounded in $H^{p}$ and $H^{q}$ respectively and tending weakly to 0 in these spaces.

Let $a \in \Sigma^{m}(\Omega)$, with $m<p+q$, then $\left(a(x, D) f_{n}, g_{n}\right)_{n}$ goes to 0 as $n$ goes to $+\infty$.

Let $t>0$, in the remainder of this paper, $\tilde{\mu}(t)$ is a $H^{1}$-microlocal defect measure of $\left(u^{n}(t)\right)_{n}, \tilde{\lambda}(t)$ is a $L^{2}$-microlocal defect measure of $\left(\partial_{t} u^{n}(t)\right)_{n}$, $\mu(t, x, \omega)$ and $\lambda(t, x, \omega)$ are in $L^{\infty}\left([0, T], \mathcal{M}^{+}\left(\Omega \times S^{d-1}\right)\right)$ and satisfy (13) and (14). We will prove using the measures $\mu$ and $\lambda$ that the measures $\tilde{\mu}(t)$ and $\tilde{\lambda}(t)$ does not depend on the extracted subsequence and they are equal to 0 on $\Omega \times S^{d-1}$, which gives Proposition 1 .

\subsection{Calculus of the measures on $\Lambda$}

Proposition 4. If $\mu_{0}(\Lambda)=\lambda_{0}(\Lambda)=0$ and $\Lambda$ is bounded, then for all $t \geq 0$ 


$$
\tilde{\mu}(t, \Lambda)=\tilde{\lambda}(t, \Lambda)=0
$$

and

$$
\mu(t, \Lambda)=\lambda(t, \Lambda)=0 .
$$

Remark 2. This proposition crucially uses the assumptions $\Lambda$ is bounded and $\mu_{0}(\Lambda)=\lambda_{0}(\Lambda)=0$. It will be interesting to study the situations where one of the previous assumptions is not fulfilled.

Proof. First we will prove that if $\mu_{0}(\Lambda)=\lambda_{0}(\Lambda)=0$ and $\Lambda$ is bounded, then for all $t \geq 0$

$$
\tilde{\mu}(t, \Lambda)=\tilde{\lambda}(t, \Lambda)=0,
$$

then we use the dominated convergence Theorem to prove the same result for the measures $\lambda$ and $\mu$.

Since $\Lambda$ is bounded, it is a compact subset of $\Omega \times S^{d-1}$. Therefore, there exists a family of smooth compactly supported functions $\psi_{\varepsilon}(x, \omega)$ such that

$$
\left\{\begin{array}{c}
0 \leq \psi_{\varepsilon}(x, \omega) \leq 1 \\
\psi_{\varepsilon}(x, \omega)=1 \text { in a neighborhood of } \Lambda \\
\psi_{\varepsilon} \underset{\varepsilon \rightarrow 0}{\longrightarrow} 1_{\Lambda} .
\end{array}\right.
$$

Let $\chi \in C_{0}^{\infty}(\mathbb{R})$ such that

$$
\left\{\begin{array}{l}
\chi(u)=0 \text { for }|u|>1 \\
\chi(u)=1 \text { for }|u|<\frac{1}{2} \\
0 \leq \chi \leq 1
\end{array}\right.
$$

and $a_{\varepsilon}$ defined by

$$
a_{\varepsilon}(x, \xi)=(1-\chi(\xi)) \psi_{\varepsilon}\left(x, \frac{\xi}{|\xi|}\right)
$$

Then $a_{\varepsilon} \in \Sigma_{c}^{0}$ and

$$
\nabla_{x} a_{\varepsilon}(x, \xi) \in \Sigma_{\Lambda}^{0}, \quad \nabla_{\xi} a_{\varepsilon}(x, \xi)=b(x, \xi)+k(x, \xi),
$$

where $b \in \Sigma_{\Lambda}^{-1}$ and $k$ is compactly supported in the variable $\xi$, then $k(x, D)$ is a compact operator of $L^{2}(\Omega)$. We define

$$
\mathcal{J}_{\varepsilon}^{n}(t)=\left(a_{\varepsilon}(x, D) \partial_{t} u^{n}(t) \mid \partial_{t} u^{n}(t)\right)+\left(a_{\varepsilon}(x, D) C(x) \nabla u^{n}(t) \mid \nabla u^{n}(t)\right) .
$$


We have for all $t \geq 0$

$$
\begin{aligned}
& \limsup _{\varepsilon \rightarrow 0} \limsup _{n \rightarrow+\infty} \mathcal{J}_{\varepsilon}^{n}(t) \\
& =\limsup _{\varepsilon \rightarrow 0} \int_{\Omega \times S^{d-1}} \psi_{\varepsilon}(x, \omega)[\tilde{\lambda}(t, d x, d \omega)+C(x) \omega \cdot \omega \tilde{\mu}(t, d x, d \omega)] \\
& =\int_{\Lambda}[\tilde{\lambda}(t, x, \omega)+C(x) \omega \cdot \omega \tilde{\mu}(t, d x, d \omega)] .
\end{aligned}
$$

Moreover if we derive (20) with respect to $t$ we obtain

$$
\frac{d}{d t} \mathcal{J}_{\varepsilon}^{n}(t)=\mathcal{J}_{1, \varepsilon}^{n}(t)+\mathcal{J}_{2, \varepsilon}^{n}(t)+\mathcal{J}_{3, \varepsilon}^{n}(t),
$$

with

$$
\begin{aligned}
\mathcal{J}_{1, \varepsilon}^{n}(t)=- & \left(a_{\varepsilon}(x, D) q(x, D)^{*} q(x, D) \partial_{t} u^{n}(t) \mid \partial_{t} u^{n}(t)\right) \\
- & \left(a_{\varepsilon}(x, D) \partial_{t} u^{n}(t) \mid q(x, D)^{*} q(x, D) \partial_{t} u^{n}(t)\right) . \\
\mathcal{J}_{2, \varepsilon}^{n}(t)= & \left(a_{\varepsilon}(x, D) \nabla \cdot\left(C(x) \nabla u^{n}(t)\right) \mid \partial_{t} u^{n}(t)\right) \\
& +\left(a_{\varepsilon}(x, D) C(x) \nabla u^{n}(t) \mid \nabla \partial_{t} u^{n}(t)\right) . \\
J_{3, \varepsilon}^{n}(t)= & \left(a_{\varepsilon}(x, D) \partial_{t} u^{n}(t) \mid \nabla \cdot\left(C(x) \nabla u^{n}(t)\right)\right) \\
& \left.+\left(a_{\varepsilon}(x, D) C(x) \nabla \partial_{t} u^{n}(t)\right) \mid \nabla u^{n}(t)\right) .
\end{aligned}
$$

We claim that for all $T>0$

$$
\limsup _{\varepsilon \rightarrow 0} \limsup _{n \rightarrow+\infty} \int_{0}^{T} J_{j, \varepsilon}^{n}(t) d t=0, \quad \forall j \in\{2,3\} .
$$

Indeed, we have :

$$
\mathfrak{J}_{2, \varepsilon}^{n}(t)=\left(\left[a_{\varepsilon}(x, D), \nabla\right] .\left(C(x) \nabla u^{n}(t)\right) \mid \partial_{t} u^{n}(t)\right) .
$$

Since $\left[a_{\varepsilon}(x, D), \nabla\right]=r(x, D)$, with $r \in \Sigma_{\Lambda}^{0}$, then

$$
\mathrm{J}_{2, \varepsilon}^{n}(t)=\left(C(x) \nabla u^{n}(t) \mid r^{*}(x, D) \partial_{t} u^{n}(t)\right) .
$$


Let $R>0$, we write

$$
\begin{array}{r}
\left(C(x) \nabla u^{n}(t) \mid r^{*}(x, D) \partial_{t} u^{n}(t)\right)=\left(C(x) \nabla u^{n}(t) \mid \psi\left(\frac{|D|}{R}\right) r^{*}(x, D) \partial_{t} u^{n}(t)\right) \\
+\left(C(x) \nabla u^{n}(t) \mid\left(1-\psi\left(\frac{|D|}{R}\right)\right) r^{*}(x, D) \partial_{t} u^{n}(t)\right)
\end{array}
$$

where $\psi$ is a cut-off function on $\mathbb{R}^{d}$ satisfying

$$
\left\{\begin{aligned}
0 \leq \psi \leq 1 & \\
\psi(x) & =0, \quad|x| \geq 1 \\
\psi(x) & =1, \quad|x| \leq 1 / 2 .
\end{aligned}\right.
$$

Since $\left(\partial_{t} u^{n}(t)\right)_{n}$ goes weakly to 0 in $L^{2}(\Omega)$ and $\psi\left(\frac{|D|}{R}\right) r^{*}(x, D)$ is a compact operator from $L^{2}(\Omega)$ into itself then

$$
\psi\left(\frac{|D|}{R}\right) r^{*}(x, D) \partial_{t} u^{n}(t) \underset{n \rightarrow+\infty}{\longrightarrow} 0 \text { in } L^{2}(\Omega) .
$$

Therefore, since $\left(\nabla u^{n}(t)\right)_{n}$ is u.b. in $L^{2}(\Omega)$, we get

$$
\forall t \in \mathbb{R}_{+},\left(C(x) \nabla u^{n}(t) \mid \psi\left(\frac{|D|}{R}\right) r^{*}(x, D) \partial_{t} u^{n}(t)\right) \underset{n \rightarrow+\infty}{\longrightarrow} 0 .
$$

On the other hand, since $r \in \Sigma_{\Lambda}^{0}$ by (19), then by Proposition $3,\left(r(x, D)^{*} \partial_{t} u^{n}\right)$ is u.b. in $L^{2}\left([0, T], H^{\alpha}(\Omega)\right)$. Which gives

$$
\begin{aligned}
\| & \left(1-\psi\left(\frac{|D|}{R}\right) r^{*}(x, D) \partial_{t} u^{n}(t)\right) \|_{L^{2}(\Omega)}^{2} \\
= & C \int_{|\xi|>\frac{R}{2}}\left(1-\psi\left(\frac{|\xi|}{R}\right)\right) \mid r^{*}\left(x,\left.\widehat{D) \partial_{t}} u^{n}(t)(\xi)\right|^{2} d \xi\right. \\
= & C\left(\int_{R \geq|\xi|>\frac{R}{2}}\left(1-\psi\left(\frac{|\xi|}{R}\right)\right) \mid r^{*}\left(x,\left.\widehat{D) \partial_{t}} u^{n}(t)(\xi)\right|^{2} d \xi\right.\right. \\
& +\int_{|\xi|>R}\langle\xi\rangle^{\alpha} \mid r^{*}\left(x,\left.\widehat{D) \partial_{t}} u^{n}(t)(\xi)\right|^{2} \frac{1}{\langle\xi\rangle^{\alpha}} d \xi\right) \\
\leq & \frac{C^{\prime}}{R^{\alpha}}\left\|r^{*}(x, D) \partial_{t} u^{n}(t)\right\|_{H^{\alpha}(\Omega)}^{2}
\end{aligned}
$$


then

$$
\begin{aligned}
& \left(C(x) \nabla u^{n}(t) \mid\left(1-\psi\left(\frac{|D|}{R}\right)\right) r^{*}(x, D) \partial_{t} u^{n}(t)\right) \\
& \leq \frac{C^{\prime}}{R^{\alpha}}\left\|\nabla u^{n}(t)\right\|_{L^{2}(\Omega)}\left\|r^{*}(x, D) \partial_{t} u^{n}(t)\right\|_{H^{\alpha}(\Omega)} .
\end{aligned}
$$

So

$$
\int_{0}^{T}\left(C(x) \nabla u^{n}(t) \mid\left(1-\psi\left(\frac{|D|}{R}\right)\right) r^{*}(x, D) \partial_{t} u^{n}(t)\right) d t \leq \frac{C^{\prime \prime}}{R^{\alpha}}
$$

By letting $R$ go to $+\infty$ we get

$$
\int_{0}^{T}\left(C(x) \nabla u^{n}(t) \mid\left(1-\psi\left(\frac{|D|}{R}\right)\right) r^{*}(x, D) \partial_{t} u^{n}(t)\right) d t \underset{n \rightarrow+\infty}{\longrightarrow} 0
$$

We conclude using $(23),(25)$ and (27) that

$$
\int_{0}^{T} \mathcal{J}_{2, \varepsilon}^{n}(t) d t \underset{n \rightarrow+\infty}{\longrightarrow} 0 \text {. }
$$

Moreover

$$
\mathcal{J}_{3, \varepsilon}^{n}(t)=\left(\left[a_{\varepsilon}(x, D), C(x) \nabla\right] . \nabla u^{n}(t) \mid \partial_{t} u^{n}(t)\right) .
$$

Since $\left[a_{\varepsilon}(x, D), C(x) \nabla\right]=r(x, D)+k(x, D)$ with $r \in \Sigma_{\Lambda}^{0}$ and $k(x, D)$ is a compact operator, then arguing as above we obtain

$$
\int_{0}^{T} \mathcal{J}_{3, \varepsilon}^{n}(t) d t \underset{n \rightarrow+\infty}{\longrightarrow} 0 .
$$

Now it remains to study the term $\mathcal{J}_{1, \varepsilon}^{n}(t)$. Since

$$
a_{\varepsilon}(x, D) q(x, D)^{*}=q(x, D)^{*} a_{\varepsilon}(x, D)+r_{1}(x, D),
$$

with $r_{1} \in \Sigma_{\Lambda}^{\alpha-1}$, and

$$
q(x, D) a_{\varepsilon}(x, D)=a_{\varepsilon}(x, D) q(x, D)+r_{2}(x, D),
$$

with $r_{2} \in \Sigma_{\Lambda}^{\alpha-1}$, then we get

$$
\begin{aligned}
\mathcal{J}_{1, \varepsilon}^{n}(t)= & -\left(a_{\varepsilon}(x, D) q(x, D) \partial_{t} u^{n}(t) \mid q(x, D) \partial_{t} u^{n}(t)\right) \\
& -\left(q(x, D) \partial_{t} u^{n}(t) \mid r_{1}^{*}(x, D) \partial_{t} u^{n}(t)\right) \\
& -\left(a_{\varepsilon}(x, D) q(x, D) \partial_{t} u^{n}(t) \mid q(x, D) \partial_{t} u^{n}(t)\right) \\
& -\left(r_{2}(x, D) \partial_{t} u^{n}(t) \mid q(x, D) \partial_{t} u^{n}(t)\right) .
\end{aligned}
$$


Since $r_{1} \in \Sigma_{\Lambda}^{\alpha-1}$, then by Proposition 3, the family $\left(r_{1}^{*}(x, D) \partial_{t} u^{n}(t)\right)_{n}$ is u.b. in $L^{2}\left([0, T], H^{1}(\Omega)\right)$. In view of the fact that $\left(q(x, D) \partial_{t} u^{n}(t)\right)_{n}$ is u.b in $L^{2}\left([0, T], L^{2}(\Omega)\right)$, we get

$$
\int_{0}^{T}\left(q(x, D) \partial_{t} u^{n}(t) \mid r_{1}^{*}(x, D) \partial_{t} u^{n}(t)\right) d t \underset{n \rightarrow+\infty}{\longrightarrow} 0
$$

For the same reasons, we obtain

$$
\int_{0}^{T}\left(r_{2}(x, D) \partial_{t} u^{n}(t) \mid q(x, D) \partial_{t} u^{n}(t)\right) d t \underset{n \rightarrow+\infty}{\longrightarrow} 0
$$

Which gives

$$
\int_{0}^{T} \mathcal{J}_{1, \varepsilon}^{n}(t) d t=-2 \int_{0}^{T}\left(a_{\varepsilon}(x, D) q(x, D) \partial_{t} u^{n}(t) \mid q(x, D) \partial_{t} u^{n}(t)\right) d t+o(1) .
$$

Besides, since $\left(q(x, D) \partial_{t} u^{n}\right)_{n}$ is u.b in $L^{2}\left([0, T], L^{2}(\Omega)\right)$ and $a_{\varepsilon}$ is a positive symbol, then thanks to the Garding inequality, the limit of the family

$$
\left(\int_{0}^{T}\left(a_{\varepsilon}(x, D) q(x, D) \partial_{t} u^{n}(t) \mid q(x, D) \partial_{t} u^{n}(t)\right)\right)_{n}
$$

is positive. Therefore we get as $n$ goes to $+\infty$ and for all $\varepsilon>0$

$$
\begin{aligned}
& \lim _{n \rightarrow+\infty} \int_{0}^{T} \mathcal{J}_{1, \varepsilon}^{n}(t) d t \\
& =-2 \lim _{n \rightarrow+\infty} \int_{0}^{T}\left(a_{\varepsilon}(x, D) q(x, D) \partial_{t} u^{n}(t) \mid q(x, D) \partial_{t} u^{n}(t)\right) d t \leq 0 .
\end{aligned}
$$

On the other hand by (21),

$$
\lim _{n \rightarrow+\infty}\left(\mathcal{J}_{\varepsilon}^{n}(T)-\mathcal{J}_{\varepsilon}^{n}(0)\right)=\lim _{n \rightarrow+\infty}\left(\sum_{i=1}^{3} \int_{0}^{T} \mathcal{J}_{i, \varepsilon}^{n}(t) d t\right) .
$$

Then by (28), (29) and (33) we get

$$
\lim _{n \rightarrow+\infty}\left(\mathcal{J}_{\varepsilon}^{n}(T)-\mathcal{J}_{\varepsilon}^{n}(0)\right)=\lim _{n \rightarrow+\infty} \int_{0}^{T} \mathcal{J}_{1, \varepsilon}^{n}(t) d t \leq 0 .
$$

Using the definition of $\tilde{\mu}$ and $\tilde{\lambda}$, we obtain

$$
\left[\int \psi_{\varepsilon}(x, \omega)[C(x) \omega \cdot \omega \tilde{\mu}(t, d x, d \omega)+\tilde{\lambda}(t, d x, d \omega)]\right]_{0}^{T} \leq 0 .
$$


As $\varepsilon$ goes to 0 , since $\mu_{0}$ and $\lambda_{0}$ are supported outside $\Lambda$, we obtain

$$
\int_{\Lambda}[C(x) \omega \cdot \omega \tilde{\mu}(T, d x, d \omega)+\tilde{\lambda}(T, d x, d \omega)] \leq 0 .
$$

then by the positivity of the measures and of the matrix $C(x)$ this gives that $\tilde{\mu}(t)$ and $\tilde{\lambda}(t)$ are equal to 0 on $\Lambda$ for all $t \geq 0$.

On the other hand for all $T>0$ and for all $\phi \in C_{0}^{\infty}([0, T])$ we have

$$
\begin{aligned}
& \limsup _{\varepsilon \rightarrow 0} \limsup _{n \rightarrow+\infty} \int_{0}^{T} \int_{\Lambda} \phi(t) \mathcal{J}_{\varepsilon}^{n}(t) d t \\
= & \limsup _{\varepsilon \rightarrow 0} \limsup _{n \rightarrow+\infty} \int_{0}^{T} \int_{\Lambda} \phi(t)\left(a_{\varepsilon}(x, D) \partial_{t} u^{n}(t) \mid \partial_{t} u^{n}(t)\right) d t \\
& +\quad \limsup _{\varepsilon \rightarrow 0} \limsup _{n \rightarrow+\infty} \int_{0}^{T} \int_{\Lambda} \phi(t)\left(a_{\varepsilon}(x, D) C(x) \nabla u^{n}(t) \mid \nabla u^{n}(t)\right) d t \\
= & \int_{0}^{T} \int_{\Lambda} \phi(t)[C(x) \omega \cdot \omega \mu(t, d x, d \omega)+\lambda(t, d x, d \omega)] d t .
\end{aligned}
$$

Since

$$
\limsup _{\varepsilon \rightarrow 0} \limsup _{n \rightarrow+\infty} \mathcal{J}_{\varepsilon}^{n}(t)=0,
$$

then using the dominated convergence theorem, we get for all $T>0$ and for all $\phi \in C_{0}^{\infty}([0, T])$

$$
\int_{0}^{T} \int_{\Lambda} \phi(t)[C(x) \omega \cdot \omega \mu(t, d x, d \omega)+\lambda(t, d x, d \omega)] d t=0 .
$$

Using again by the positivity of the measures $\mu$ and $\lambda$ and of the matrix $C(x)$, this gives that $\mu$ and $\lambda$ are equal to 0 on $\Lambda$ and this complete the proof of Proposition 4.

\subsection{Calculus of the measures $\mu$ and $\lambda$ outside $\Lambda$}

Proposition 5. In $\{t>0\}$, we have

$$
\lambda=0 \quad \text { and } \quad \mu=0 \quad \text { outside } \Lambda .
$$

Proof. The result $\lambda=0$ on $\Lambda^{c}$ is a consequence of Proposition 3. Indeed, since for all $T>0$ and for all $a \in \Sigma_{\Lambda}^{0},\left(a(x, D) \partial_{t} u^{n}\right)_{n}$ is u.b. in $L^{2}\left([0, T], H^{\alpha}(\Omega)\right)$ 
then by the Rellich Lemma

$$
\left(a(x, D) \partial_{t} u^{n}(t) \mid \partial_{t} u^{n}(t)\right) \underset{n \rightarrow+\infty}{\longrightarrow} 0 .
$$

Therefore using the dominated convergence theorem, we get that $\lambda=0$ on $\Lambda^{c}$.

Let us prove that $\mu=0$ on $\Lambda^{c}$. Let $\phi \in C_{0}^{\infty}\left(\mathbb{R}_{+}^{*}\right), a \in \Sigma_{\Lambda}^{0}$ be a real-valued symbol and $\left(L^{n}\right)_{n}$ the sequence defined by :

$$
L^{n}=\int \phi(t)\left(a(x, D) u^{n}(t) \mid \partial_{t}^{2} u^{n}(t)\right) d t .
$$

By integration by parts, we obtain

$$
L^{n}=-\int \phi^{\prime}(t)\left(a(x, D) u^{n}(t) \mid \partial_{t} u^{n}(t)\right) d t-\int \phi(t)\left(a(x, D) \partial_{t} u^{n}(t) \mid \partial_{t} u^{n}(t)\right) d t .
$$

By Rellich Lemma, we have

$$
\left(a(x, D) u^{n}(t) \mid \partial_{t} u^{n}(t)\right) \underset{n \rightarrow+\infty}{\longrightarrow} 0,
$$

then

$$
L^{n} \underset{n \rightarrow+\infty}{\longrightarrow}-\iint \phi(t) a_{0}(x, \omega) \lambda(t, d x, d \omega) d t
$$

Since $\lambda=0$ on $\Lambda^{c}$, we obtain

$$
L^{n} \underset{n \rightarrow+\infty}{\longrightarrow} 0
$$

Besides, we write

$$
L^{n}=L_{1}^{n}+L_{2}^{n},
$$

with

$$
\begin{aligned}
& L_{1}^{n}=\int \phi(t)\left(a(x, D) u^{n}(t) \mid \nabla \cdot\left(C(x) \nabla u^{n}(t)\right)\right) d t . \\
& \left.L_{2}^{n}=-\int \phi(t)\left(a(x, D) u^{n}(t) \mid q(x, D)^{*} q(x, D) \partial_{t} u^{n}(t)\right)\right) d t .
\end{aligned}
$$

By the definition of $\mu$ we have

$$
L_{1}^{n} \underset{n \rightarrow+\infty}{\longrightarrow}-\int \phi(t) C(x) \omega \cdot \omega a_{0}(x, \omega) \mu(t, d x, d \omega) d t .
$$

Using symbolic calculus, we get 


$$
\left.\left(q(x, D)^{*} q(x, D) \circ a(x, D)\right)^{*}=q(x, D)^{*} q(x, D) \circ a(x, D)\right)+r(x, D),
$$

with $r(x, D) \in \Sigma_{\Lambda}^{2 \alpha-1}$. Therefore, the same argument as the one developed in the proof of Proposition 4, gives for $T>0$

$$
\int \phi(t)\left(r(x, D) \partial_{t} u^{n}(t) \mid u^{n}(t)\right) d t \underset{n \rightarrow+\infty}{\longrightarrow} 0
$$

so we have

$$
L_{2}^{n}=-\int \phi(t)\left(q(x, D)^{*} q(x, D) a(x, D) u^{n}(t) \mid \partial_{t} u^{n}(t)\right) d t+o(1) .
$$

On the other hand

$$
\begin{aligned}
& \left(q(x, D)^{*} q(x, D) a(x, D) u^{n}(t) \mid \partial_{t} u^{n}(t)\right) \\
= & \left(q(x, D) a(x, D) u^{n}(t) \mid q(x, D) \partial_{t} u^{n}(t)\right),
\end{aligned}
$$

since $q(x, D) a(x, D) u^{n}(t)$ is u.b. in $L^{2}\left([0, T], L^{2}(\Omega)\right)$ and $\left(q(x, D) \partial_{t} u^{n}(t)\right)_{n}$ is u.b. in $L^{2}\left([0, T], H^{\alpha}(\Omega)\right)$, we get

$$
L_{2}^{n} \underset{n \rightarrow+\infty}{\longrightarrow} 0
$$

and

$$
\int \phi(t) C(x) \omega \cdot \omega a_{0}(x, \omega) \mu(t, d x, d \omega) d t=0,
$$

which gives Proposition 5 .

\subsection{Calculus of the measures $\tilde{\mu}$ and $\tilde{\lambda}$ outside $\Lambda$}

Proposition 6. Let $T>0$, there exist a subsequence $n_{k}$ and a map $t \mapsto \tilde{\nu}(t)$ from $[0, T]$ into the set of positive Radon measures on $\Omega \times S^{d-1}$, such that for all $t \in[0, T]$ and for all $a \in \Sigma_{\Lambda}^{0}$, we have

$$
\begin{aligned}
& \left(a(x, D) \partial_{t} u^{n_{k}}(t) \mid \partial_{t} u^{n_{k}}(t)\right)+\left(a(x, D) C(x) \nabla u^{n_{k}}(t) \mid \nabla u^{n_{k}}(t)\right) \\
& \underset{k \rightarrow+\infty}{\longrightarrow} \int_{\Omega \times S^{d-1}} a_{0}(x, \omega) \tilde{\nu}(t, d x, d \omega)
\end{aligned}
$$

where the convergence is uniform on $[0, T]$ and

$$
\tilde{\nu}(t, x, \omega)=\tilde{\lambda}(t, x, \omega)+C(x) \omega \omega \tilde{\mu}(t, x, \omega) .
$$


Proof. Let $a \in \Sigma_{\Lambda}^{0}$, we denote by

$$
g^{n}(t)=\left(a(x, D) \partial_{t} u^{n}(t) \mid \partial_{t} u^{n}(t)\right)+\left(a(x, D) C(x) \nabla u^{n}(t) \mid \nabla u^{n}(t)\right) .
$$

We have

$$
\frac{d}{d t} \partial^{n}(t)=\mathcal{J}_{1}^{n}(t)+\mathcal{J}_{2}^{n}(t)+\mathcal{J}_{3}^{n}(t)
$$

with

$$
\begin{aligned}
\partial_{1}^{n}(t)= & -\left(a(x, D) q(x, D)^{*} q(x, D) \partial_{t} u^{n}(t) \mid \partial_{t} u^{n}(t)\right) \\
& -\left(a(x, D) \partial_{t} u^{n}(t) \mid q(x, D)^{*} q(x, D) \partial_{t} u^{n}(t)\right) .
\end{aligned}
$$

$$
\begin{aligned}
& \partial_{2}^{n}(t)=\left(a(x, D) \nabla \cdot\left(C(x) \nabla u^{n}(t)\right) \mid \partial_{t} u^{n}(t)\right)+\left(a(x, D) C(x) \nabla u^{n}(t) \mid \nabla \partial_{t} u^{n}(t)\right) . \\
& \left.\partial_{3}^{n}(t)=\left(a(x, D) \partial_{t} u^{n}(t) \mid \nabla \cdot\left(C(x) \nabla u^{n}(t)\right)\right)+\left(a(x, D) C(x) \nabla \partial_{t} u^{n}(t)\right) \mid \nabla u^{n}(t)\right) .
\end{aligned}
$$

We claim that for all $0 \leq T \leq T^{\prime}$,

$$
\lim _{n \rightarrow+\infty} \int_{T}^{T^{\prime}} g_{j}^{n}(t) d t=0, \quad \forall j \in\{2,3\} .
$$

Indeed, we have :

$$
\mathrm{J}_{2}^{n}(t)=\left([a(x, D), \nabla] \cdot\left(C(x) \nabla u^{n}(t)\right) \mid \partial_{t} u^{n}(t)\right) .
$$

Since $[a(x, D), \nabla]=r(x, D)$, with $r \in \Sigma_{\Lambda}^{0}$, the sequence $\left(r(x, D)^{*} \partial_{t} u^{n}\right)_{n}$ is u.b. in $L^{2}\left(\left[T, T^{\prime}\right], H^{1}(\Omega)\right)$. Besides, using that $\left(\nabla u^{n}\right)_{n}$ is u.b. in $L^{2}\left(\left[T, T^{\prime}\right], L^{2}(\Omega)\right)$, we get

$$
\int_{T}^{T^{\prime}} \partial_{2}^{n}(t) d t \underset{n \rightarrow+\infty}{\longrightarrow} 0 .
$$

Moreover

$$
\partial_{3}^{n}(t)=\left([a(x, D), C(x) \nabla] \cdot\left(\nabla u^{n}(t) \mid \partial_{t} u^{n}(t)\right) .\right.
$$

Since $[a(x, D), C(x) \nabla]=\tilde{r}(x, D)$ with $\tilde{r} \in \Sigma_{\Lambda}^{0}$, then arguing as above, we obtain

$$
\int_{T}^{T^{\prime}} \partial_{3}^{n}(t) d t \underset{n \rightarrow+\infty}{\longrightarrow} 0 .
$$


On the other hand,

$$
\begin{aligned}
\int_{T}^{T^{\prime}} \partial_{1}^{n}(t) d t= & -\int_{T}^{T^{\prime}}\left(a(x, D) q(x, D)^{*} q(x, D) \partial_{t} u^{n}(t) \mid \partial_{t} u^{n}(t)\right) d t \\
& -\int_{T}^{T^{\prime}}\left(a(x, D) \partial_{t} u^{n}(t) \mid q(x, D)^{*} q(x, D) \partial_{t} u^{n}(t)\right) d t
\end{aligned}
$$

By symbolic calculus,

$$
a(x, D) q(x, D)^{*}=q(x, D)^{*} a(x, D)+\tilde{a}(x, D),
$$

with $\tilde{a} \in \Sigma_{\Lambda}^{\alpha-1}$. Then

$$
\begin{aligned}
& \left(a(x, D) q(x, D)^{*} q(x, D) \partial_{t} u^{n}(t) \mid \partial_{t} u^{n}(t)\right) \\
& =\left(a(x, D) q(x, D) \partial_{t} u^{n}(t) \mid q(x, D) \partial_{t} u^{n}(t)\right) \\
& +\left(\tilde{a}(x, D) q(x, D) \partial_{t} u^{n}(t) \mid \partial_{t} u^{n}(t)\right) .
\end{aligned}
$$

Since $\left(q(x, D) \partial_{t} u^{n}(t)\right)_{n}$ is u.b. in $L^{2}\left(\left[T, T^{\prime}\right], L^{2}(\Omega)\right)$, then

$$
\left(\left(\tilde{a}(x, D) q(x, D) \partial_{t} u^{n}(t)\right)_{n} \text { is u.b. in } L^{2}\left(\left[T, T^{\prime}\right], H^{1-\alpha}(\Omega)\right)\right.
$$

which gives ,

$$
\int_{T}^{T^{\prime}}\left(\tilde{a}(x, D) q(x, D) \partial_{t} u^{n}(t) \mid q(x, D) \partial_{t} u^{n}(t)\right) d t \underset{n \rightarrow+\infty}{\longrightarrow} 0
$$

Then

$$
\begin{aligned}
& \int_{T}^{T^{\prime}}\left(a(x, D) q(x, D)^{*} q(x, D) \partial_{t} u^{n}(t) \mid \partial_{t} u^{n}(t)\right) d t \\
& =\int_{T}^{T^{\prime}}\left(a(x, D) q(x, D) \partial_{t} u^{n}(t) \mid q(x, D) \partial_{t} u^{n}(t)\right) d t+o(1)
\end{aligned}
$$


Similarly, we prove that

$$
\begin{aligned}
& \int_{T}^{T^{\prime}}\left(a(x, D) \partial_{t} u^{n}(t) \mid q(x, D)^{*} q(x, D) \partial_{t} u^{n}(t)\right) d t \\
& =\int_{T}^{T^{\prime}}\left(a(x, D) q(x, D) \partial_{t} u^{n}(t) \mid q(x, D) \partial_{t} u^{n}(t)\right) d t+o(1) .
\end{aligned}
$$

Hence

$$
\int_{T}^{T^{\prime}} \partial_{1}^{n}(t) d t=-2 \int_{T}^{T^{\prime}}\left(a(x, D) q(x, D) \partial_{t} u^{n}(t) \mid q(x, D) \partial_{t} u^{n}(t)\right) d t+o(1) .
$$

Since $\left(q(x, D) \partial_{t} u^{n}(t)\right)_{n}$ is u.b. in $L^{2}\left(\left[0, T^{\prime}\right], L^{2}(\Omega)\right)$, there exists a positive constant $C_{a}\left(T^{\prime}\right)$ which depends on $T^{\prime}$ and on the operator $a(x, D)$ such that

$$
\begin{aligned}
\left|\int_{T}^{T^{\prime}} \partial_{1}^{n}(t) d t\right| & \leq C_{a}\left(T^{\prime}\right)\left|T-T^{\prime}\right|+o(1) \\
& \leq C_{a}^{\prime}\left(T^{\prime}\right)\left|T-T^{\prime}\right|
\end{aligned}
$$

By (38), (40) and (45) we conclude that

$$
\left|g^{n}(T)-g^{n}\left(T^{\prime}\right)\right| \leq C_{a}^{\prime \prime}\left(T^{\prime}\right)\left|T-T^{\prime}\right| .
$$

Therefore the sequence $\left(g^{n}\right)_{n}$ of $\mathcal{C}\left(\left[0, T^{\prime}\right], \mathbb{C}\right)$ is equicontinuous, and since for all fixed $t$ of $\left[0, T^{\prime}\right],\left(g^{n}(t)\right)_{n}$ is bounded, then Ascoli Theorem yields the existence of a subsequence $\left(g^{n_{k}}\right)_{k}$ which converges uniformly on $\left[0, T^{\prime}\right]$. Hence if $\tilde{\mu}(t)$ is a $H^{1}$-microlocal defect measure of $\left(u^{n}(t)\right)_{n}$ and $\tilde{\lambda}(t)$ is a $L^{2}$-microlocal defect measure of $\left(\partial_{t} u^{n}(t)\right)_{n}$, we get

$$
\begin{aligned}
\left(a(x, D) \partial_{t} u^{n_{k}}(t) \mid \partial_{t} u^{n_{k}}(t)\right) & +\left(a(x, D) C(x) \nabla u^{n_{k}}(t) \mid \nabla u^{n_{k}}(t)\right) \\
& \longrightarrow \underset{k \rightarrow+\infty}{\longrightarrow} \int_{\Omega \times S^{d-1}} a_{0}(x, \omega) \tilde{\nu}(t, d x, d \omega)
\end{aligned}
$$

where the convergence is uniform on $\left[0, T^{\prime}\right]$ and

$$
\tilde{\nu}(t, x, \omega)=\tilde{\lambda}(t, x, \omega)+C(x) \omega \omega \tilde{\mu}(t, x, \omega) .
$$

By considering a dense countable subset of $\Sigma_{\Lambda}^{0}$ and by diagonal extraction, we may assume that the subsequence $\left(\mathcal{J}^{n_{k}}\right)_{k}$ is the same for any $a \in \Sigma_{\Lambda}^{0}$, which gives Proposition 6 . 
Proposition 7. In $\{t>0\}$, we have

$$
\tilde{\lambda}=0 \quad \text { and } \quad \tilde{\mu}=0 \text { on } \Lambda^{c} .
$$

Proof. Let $a \in \Sigma_{\Lambda}^{0}, T>0$ and $\phi \in C_{0}^{\infty}([0, T])$, by Proposition 6 , there exists a subsequence $n_{k}$ and a map $t \mapsto \tilde{\nu}(t)$ from [0,T] into the set of the positive Radon measures on $\Omega \times S^{d-1}$ such that for all $t \in[0, T]$, we have

$$
\begin{array}{r}
\int_{0}^{T} \phi(t)\left[\left(a(x, D) \partial_{t} u^{n_{k}}(t) \mid \partial_{t} u^{n_{k}}(t)\right)+\left(a(x, D) C(x) \nabla u^{n_{k}}(t) \mid \nabla u^{n_{k}}(t)\right)\right] d t \\
\underset{k \rightarrow+\infty}{\longrightarrow} \int_{0}^{T} \int_{\Omega \times S^{d-1}} a_{0}(x, \omega) \tilde{\nu}(t, d x, d \omega) d t
\end{array}
$$

where

$$
\tilde{\nu}(t, x, \omega)=\tilde{\lambda}(t, x, \omega)+C(x) \omega \cdot \omega \tilde{\mu}(t, x, \omega) .
$$

On the other hand, we have by the definition of $\mu$ and $\lambda$,

$$
\begin{aligned}
\int_{0}^{T} \phi(t)\left[\left(a(x, D) \partial_{t} u^{n_{k}}(t) \mid \partial_{t} u^{n_{k}}(t)\right)\right. & \left.+\left(a(x, D) C(x) \nabla u^{n_{k}}(t) \mid \nabla u^{n_{k}}(t)\right)\right] d t \\
\underset{k}{\longrightarrow} \rightarrow+\infty & \int_{0}^{T} \int_{\Lambda^{c}} \phi(t) a_{0}(x, \omega) \nu(d t, d x, d \omega)
\end{aligned}
$$

where

$$
\nu(t, x, \omega)=\lambda(t, x, \omega)+C(x) \omega \cdot \omega \mu(t, x, \omega) .
$$

Since in $\{t>0\}$

$$
\lambda=0 \quad \text { and } \quad \mu=0 \quad \text { on } \Lambda^{c},
$$

then by ( 49$)$, we obtain in $\{t>0\}$

$$
\tilde{\nu}(t, x, \omega)=0 .
$$

By the positivity of the measures and of the matrix $C(x)$, this gives that in $\{t>0\}, \tilde{\lambda}(t)$ and $\tilde{\mu}(t)$ are equal to 0 on $\Lambda^{c}$ and this completes the proof of Proposition 7.

\subsection{Proof of Proposition 2}

Let $\chi \in C_{0}^{\infty}\left(\mathbb{R}_{+}^{*}\right)$ and $\phi \in C_{0}^{\infty}(\Omega)$. By Theorem 1, we obtain

$$
\int_{\mathbb{R}_{+} \times \Omega} \chi^{\prime}(t) \phi(x) e^{n}(t, x) d t d x \underset{n \rightarrow+\infty}{\longrightarrow} 0
$$


and in the other hand, by integration by parts we get :

$$
\int_{\mathbb{R}_{+} \times \Omega} \chi^{\prime}(t) \phi(x) e^{n}(t, x) d t d x=-\int_{\mathbb{R}_{+} \times \Omega} \chi(t) \phi(x) \frac{d}{d t}\left(e^{n}(t, x)\right) d t d x .
$$

Since $C$ is symmetric, we have

$$
\frac{d}{d t} e^{n}(t, x)=2 \partial_{t}^{2} u^{n} \partial_{t} u^{n}+2 C(x) \nabla \partial_{t} u^{n} \cdot \nabla u^{n}
$$

Using equation (1), we obtain

$$
\frac{d}{d t} e^{n}(t, x)=2 \nabla \cdot\left(C(x) \nabla u^{n} \partial_{t} u^{n}\right)-2 q(x, D)^{*} q(x, D) \partial_{t} u^{n} \partial_{t} u^{n} .
$$

Therefore

$$
\begin{aligned}
\int_{\mathbb{R}_{+} \times \Omega} \chi^{\prime}(t) \phi(x) e^{n}(t, x) d t d x & =2 \int_{\mathbb{R}_{+} \times \Omega} \chi(t) \nabla \phi(x) \cdot C(x) \nabla u^{n}(t, x) \partial_{t} u^{n}(t, x) d t d x \\
& +2 \int_{\mathbb{R}_{+} \times \Omega} \chi(t) \phi(x) q(x, D)^{*} q(x, D) \partial_{t} u^{n} \partial_{t} u^{n} d t d x .
\end{aligned}
$$

Using Proposition 1, we have $\left(\partial_{t} u^{n}(t)\right)_{n}$ goes to 0 strongly in $L_{l o c}^{2}(\Omega)$, so

$$
\begin{aligned}
& \lim _{n \rightarrow+\infty}\left[\int_{\mathbb{R}_{+} \times \Omega} \chi^{\prime}(t) \phi(x) e^{n}(t, x) d t d x\right] \\
& =\lim _{n \rightarrow+\infty}\left[2 \int_{\mathbb{R}_{+} \times \Omega} \chi(t) \phi(x) q(x, D)^{*} q(x, D) \partial_{t} u^{n} \partial_{t} u^{n} d t d x\right] .
\end{aligned}
$$

By (52) and using the fact that

$$
\begin{gathered}
\lim _{n \rightarrow+\infty}\left[2 \int_{\mathbb{R}_{+} \times \Omega} \chi(t) \phi(x) q(x, D)^{*} q(x, D) \partial_{t} u^{n} \partial_{t} u^{n} d t d x\right]= \\
\lim _{n \rightarrow+\infty}\left[2 \int_{\mathbb{R}_{+} \times \Omega} \chi(t) \phi(x) q(x, D) \partial_{t} u^{n} q(x, D) \partial_{t} u^{n} d t d x\right],
\end{gathered}
$$

we get Proposition 2 . 


\section{References}

[1] S. Alinhac, P. Gérard, Opérateurs pseudo-différentiels et théorème de Nash-Moser, Inter Éditions du CNRS, Meudon, France, 1991.

[2] A. Atallah-Baraket, C. Fermanian Kammerer, High frequency analysis of solutions to the equation of viscoelasticity of Kelvin-Voight, J. Hyperbolic. Differ. Equ. 1 (2004), 789-812.

[3] M.A. Ayadi, A. Bchatnia, M. Hamouda, S. Messaoudi, General decay in a Timoshenko-type system with thermoelasticity with second sound, Adv. Nonlinear Anal. 4 (2015), 263-284.

[4] D. Blanchard, O. Guib, Existence of a solution for a nonlinear system in thermo-viscoelasticity, Adv. Differential Equations 5 (2000), 1221-1252.

[5] B. Bougherara, J. Giacomoni, Existence of mild solutions for a singular parabolic equation and stabilization, Adv. Nonlinear Anal. 4 (2015), 123134.

[6] D. Brandon, I. Fonseca and P. Swart, Dynamics and oscillatory microstructure in a model of displacive phase transformations, Progress in partial differential equations: the Metz surveys, 3, 130144, Pitman Res. Notes Math. Ser., 314, Longman Sci. Tech., Harlow, 1994.

[7] N. Burq, Mesures semi-classique et mesures de défaut, Sminaire N. Bourbaki, (1996-1997), exp. $n^{\circ}$ 826, 167-195.

[8] N. Burq, G. Lebeau, Mesures de défaut de compacité, application au système de Lamé, Ann. Scient. Ec. Norm. Sup. 34 (2001), 817-870.

[9] E. Cordero, F. Nicola and L. Rodino, Microlocal analysis and applications, Advances in pseudo-differential operators, 117, Oper. Theory Adv. Appl., 155, Birkhuser, Basel, 2004.

[10] C. Do, On the Dynamic Deformation of a Bar against an Obstacle. Variational Methods in the Mechanics of Solids, ed. S. Nemat Nasser, Northwestern University, USA ( Pergamon Press, 1978), 237-241.

[11] G. Francfort, Introduction to H-Measures and their applications , Progress in Nonlinear Differential Equations and Their Applications, Vol. 68, 85-110, Birkhuser Verlag Basel / Switzerland. 
[12] G. Francfort, P. Gérard, The wave equation on a thin domain : Energy Density and observability, Journal of Hyperbolic Differential Equation 1 (2004), 351-366.

[13] G. Francfort, F. Murat, Oscillations and energy densities in the wave equation, Comm. Partial Differential Equations 17 (1992), 1785-1865.

[14] P. Gérard, Microlocal defect measures, Commun. Partial Differential Equations, 16 (1991), 1761-1794.

[15] P. Gérard, Oscillations and concentration effects in semilinear dispersive wave equations, J. Funct. Anal. 133 (1996), 50-68.

[16] M. Ghergu, V. Rădulescu, Nonlinear PDEs. Mathematical models in biology, chemistry and population genetics, Springer Monographs in Mathematics, Springer, Heidelberg, 2012.

[17] M. S. Joshi, Introduction to Pseudo-Differential operators, arXiv : 9906155v1, (1999).

[18] J. U. Kim, On the local regularity of solutions in linear viscoelasticity of several space dimensions , Trans. Amer . Math. Soc. 346 (1994), 359-398.

[19] G. Lebeau, Equations des ondes amorties, Séminaire de l'école polytechnique, Expos XV (1994).

[20] J. Le Rousseau, Analyse microlocale et semi classique. Applications au prolongement unique, et au contrôle des ondes et de la chaleur, unpublished manuscript.

[21] A. Petrov, M. Schatzman, Viscolastodynamique monodimensionnelle avec conditions de Signorini, C.R. Acad. Sci. Paris Ser. I 334 (2002), 983-988.

[22] M. Petrini, Behaviour of the energy density associated to a KelvinVoight model in viscoelasticity, Asymptot. Anal 34 (2003), 261-273.

[23] V. Rădulescu, D. Repovš, Partial differential equations with variable exponents. Variational methods and qualitative analysis, Monographs and Research Notes in Mathematics, CRC Press, Boca Raton, FL, 2015.

[24] L. Tartar, H-measures, a new approach for studying Homogenisation, oscillations and concentration effects in partial differential equations, Proc. Roy. Soc, Edinburgh Sect. A 115 (1990), 193-230. 
ANALYSIS OF THE ENERGY DECAY OF A VISCOELASTICITY TYPE EQUATION

[25] E. Wigner, On the quantum correction for thermodynamic equilibrium, Phys. Rev. 40 (1934), 742-759.

Amel ATALLAH-BARAKET,

Department of Mathematics,

University of Tunis El Manar,

Faculty of Sciences of Tunis, 1060, Tunis, Tunisie.

Email: amel.atallah@fst.rnu.tn

Maryem Trabelsi,

Department of Mathematics,

University of Tunis El Manar,

Faculty of Sciences of Tunis, 1060, Tunis, Tunisie.

Email: trabelsi.maryem@gmail.com 